\title{
Treinamento de intubação orotraqueal na pandemia por coronavírus: aplicação da Prática Deliberada em Ciclos Rápidos
}

\section{Orotraqueal intubation training in the coronavirus pandemic: application of the rapid cycle deliberate practice}

\author{
Rodrigo Pereira Diaz André1 (1) |diaz.rodrigo@globo.com \\ Hudson Carmo de Oliveira' (D) hudoliver@hotmail.com \\ Glauber Gouvêa' (D) glauber.gouvea@gmail.com \\ Fátima Carneiro Fernandes' (1) fatimacfernandes@gmail.com \\ Ingrid Régia Lopes Jerônimo' (1) ingridregia@hucff.ufrj.br \\ Juliana Faria Campos ${ }^{1}$ (D) jujufariacampos@yahoo.com.br
}

\begin{abstract}
RESUMO
Introdução: A pandemia por coronavírus revelou a necessidade de intubação orotraqueal de forma segura não apenas para o paciente, mas igualmente para os profissionais envolvidos no procedimento. Para isso, treinamentos e revisões de técnicas se tornam necessários. Objetivo: Este artigo tem por objetivos propor a aplicação da estratégia de Prática Deliberada em Ciclos Rápidos (PDCR) para treinamento de anestesiologistas na intubação orotraqueal em pessoas confirmadas ou suspeitas com Covid-19 e apresentar um guia para aplicação dessa estratégia nessa conjuntura.
\end{abstract}

Método: Trata-se de estudo metodológico que apresenta aspectos teóricos e operacionais para a aplicação da PDCR e um guia de aplicação construído a partir da busca de evidências publicadas em periódicos e recomendações oficiais divulgadas pelos órgãos vinculados à área da saúde brasileira e internacional.

Resultado: Os principais aspectos teóricos relatados são concernentes aos três princípios que baseiam a PDCR: maximização do tempo em prática deliberada, feedback direcionado e segurança psicológica explícita. Quanto aos aspectos operacionais, destaca-se que o treinamento deve ser realizado com o máximo de seis pessoas. Deve-se interromper o erro, fornecer um feedback prescritivo e pedir que a tarefa seja realizada novamente até atingir a maestria. Quanto às especificidades técnicas do procedimento, apresenta-se um guia de aplicação da PDCR com a sequência para o adequado manuseio de vias aéreas de pacientes hipoxêmicos suspeitos e positivos para Covid-19.

Conclusão: A estratégia instrucional estudada mostra ser propícia a treinar com maestria os profissionais que realizarão o procedimento de intubação orotraqueal no enfrentamento das formas graves da Covid-19, visando minimizar o risco de contaminação.

Palavras-chave: Infecção por Coronavírus; Intubação Intratraqueal; Pandemia; Treinamento por Simulação.

\begin{abstract}
Introduction: the coronavirus pandemic disclosed the need for safe orotracheal intubation not only for the patient, but also for the professionals involved in the procedure. Therefore, training and technique reviews became necessary.

Objective: this article aims to propose the application of the Rapid Cycle Deliberate Practice (RCDP) strategy for the training of anesthesiologists in orotracheal intubation in people with confirmed or suspected COVID-19 and to present a guide for the application of this strategy in this situation.

Method: This is a methodological study presenting the theoretical and operational aspects for the Rapid Cycle Deliberate Practice application and a guide constructed from the search for evidence published in journals and official recommendations published by the institutions linked to the Brazilian and international health area.

Results: the main theoretical aspects reported are related to the three principles on which Rapid Cycle Deliberate Practice is based: maximizing time in deliberate practice, targeted feedback, and explicit psychological security. As for the operational aspects, it is highlighted that the training must be carried out with a maximum of six people. An error must be interrupted, prescriptive feedback must be given, and the task must be performed again until the participant reaches mastery. As for the procedure technical specificity, a guide to the application of the rapid cycle deliberate practice is presented with the sequence for the adequate airway management of hypoxemic patients with suspected or confirmed COVID-19 infection.
\end{abstract}

Conclusion: it is concluded that the assessed instructional strategy showed to be promising for the training with mastery learning in all professionals who will perform the procedure of orotracheal intubation while facing the severe forms of COVID-19, minimizing the risk of contamination.

Keywords: Coronavirus Infections; Intratracheal Intubation; Pandemics; Simulation Training.

${ }^{1}$ Universidade Federal do Rio de Janeiro, Rio de Janeiro, Rio de Janeiro, Brasil.

Editora-chefe: Rosiane Viana Zuza Diniz.

Editor associado: Kristopherson Lustosa Augusto.

Recebido em 21/01/21; Aceito em 29/04/21.

Avaliado pelo processo de double blind review. 


\section{INTRODUÇÃO}

A Organização Mundial da Saúde, em 11 de março de 2020, declarou que a doença pelo novo coronavírus, denominada coronavirus disease 2019 (Covid-19), tornou-se uma pandemia. Em maio de 2020, as estatísticas mostravam quase 4,5 milhões de casos confirmados e 302.059 mortes em mais de 210 países ${ }^{1}$. Dados indicam que de $14 \%$ a $20 \%$ dos casos infectados pelo vírus severe acute respiratory syndrome-related coronavirus 2 (Sars-CoV-2) podem evoluir para a síndrome respiratória aguda grave (Sars) ${ }^{2,3}$. Isso posto, a necessidade de intubação orotraqueal foi apontada em 2,3\% de 1.099 pacientes com Covid-19 confirmados, em coorte de 552 hospitais em 30 províncias chinesas ${ }^{2}$ : em $47 \%$ de 36 pacientes internados em unidade de terapia intensiva (UTI) no Hospital Zhongnan ${ }^{3}$ e em $42 \%$ de 52 pacientes admitidos em unidade intensiva no Hospital Jin Yin-tan, ambos em Wuhan 4 .

Dada a natureza altamente contagiosa do Sars-CoV-2 e sua transmissão por gotícula, e potencial aerossolização em alguns procedimentos ${ }^{5}$, a intubação de vias aéreas traz um alto risco de contaminação para profissionais envolvidos na ação. A diretriz da sociedade de anestesiologia chinesa relata a contaminação de inúmeros anestesiologistas durante procedimentos de intubação orotraqueal em indivíduos confirmados com a Covid-19, embora poucos dados estatísticos sejam conhecidos na atual pandemia ${ }^{6}$. Contudo, sabe-se que, durante o surto de Sars em 2002, 21\% dos indivíduos infectados globalmente eram profissionais de saúde ${ }^{7}$. Dados de um estudo do Canadá revelam que, de 251 casos de Sars confirmados, $43 \%$ foram em profissionais da área da saúde, o que leva à reflexão sobre o potencial de contaminação desses agentes apesar de aparente paramentação adequada?.

O manuseio de vias aéreas desses pacientes no contexto de uma pandemia implica desafios distintos que geram riscos adicionais tanto para os pacientes quanto para os profissionais de saúde. Em relação aos pacientes, há risco de terem uma via aérea difícil e consequente intubação complicada, já que, em vigência de insuficiência respiratória, podem apresentar mecanismos compensatórios ventilatórios já esgotados. Entre os profissionais de saúde envolvidos, o risco aumentado de infecção cruzada representa desafio técnico e psicológico na realização da tarefå .

Pesquisas demonstram que a não atualização dos profissionais e a ausência de prática repetida resultam, ao longo do tempo, em desvios de técnicas ou habilidades estagnadas ${ }^{9}$. Além disso, os intervalos de retenção, que são períodos prolongados sem prática de determinada habilidade, resultam em declínio de performance ${ }^{9}$. Assim, a realização de treinamentos com todos os profissionais envolvidos na assistência com pacientes com Covid-19 é de extrema relevância ${ }^{10}$. Os treinamentos, incluindo colocação e retirada de equipamentos de proteção individual (EPI) e testes de simulação dos processos para casos críticos e não críticos, além de gerenciamento de vias aéreas, devem ser realizados para identificar problemas e lacunas do sistema. Os serviços de saúde devem garantir que sejam fornecidos recursos adequados (tempo e equipamento da equipe) para treinamento e simulação de cenários de manuseio de vias aéreas em preparação para a Covid-19 ${ }^{11}$.

Acredita-se que o preparo adequado do profissional para a realização do procedimento pode minimizar as chances de contaminação e melhorar sua performance. Um estudo realizado em dois hospitais de Wuhan, na China, analisou uma série de 202 pacientes com Covid-19 submetidos à intubação traqueal por anestesiologistas. $O$ estudo relata taxa zero de transmissão do vírus nos profissionais que realizaram intubação, devido ao investimento em práticas de treinamento educacional para a colocação e retirada adequada de EPI e o monitoramento do procedimento. Ressalta ainda que não houve evidência de infecção cruzada nesses profissionais mesmo após 14 dias do procedimento, sugere um treinamento individualizado e cogita a possibilidade de simulação antes de realizarem a intubação em pessoas com Covid-198.

Nessa perspectiva, uma estratégia de simulação inovadora cujo objetivo é melhorar a performance dos participantes pode ser útil nesse cenário. Denominada de Prática Deliberada em Ciclos Rápidos (PDCR), a estratégia promove a repetição de tarefas até a obtenção da habilidade com maestria, e, para tal, os participantes são acompanhados por um instrutor responsável pelo fornecimento de feedbacks baseados em evidências ${ }^{12}$. Assim, entende-se que a PDCR é uma estratégia instrucional de simulação centrada no aprendiz passível de aplicação para a capacitação e o treinamento com maestria de anestesiologistas no procedimento de intubação orotraqueal, assim como na paramentação e desparamentação de EPI, a fim de atender à atual demanda pandêmica ${ }^{13}$.

Nesse sentido, este artigo tem por objetivos propor a aplicação da estratégia de PDCR para treinamento de anestesiologistas na intubação orotraqueal em pessoas confirmadas ou suspeitas com Covid-19 e apresentar um guia para aplicação dessa estratégia nessa conjuntura.

\section{MATERIAIS E MÉTODOS}

Trata-se de um estudo metodológico que apresenta uma estratégia instrucional de simulação para ser utilizada como método de treinamento de anestesiologistas na intubação orotraqueal em pessoas confirmadas ou suspeitas com Covid-19. O estudo foi estruturado em duas etapas. $\mathrm{Na}$ primeira etapa, realizou-se busca exploratória de evidências 
sobre as principais atualizações para a intubação orotraqueal no contexto da pandemia. Consideraram-se as principais diretrizes e recomendações oficiais divulgadas pelos órgãos vinculados à área da saúde brasileira e internacional: Organização Mundial da Saúde, Ministério da Saúde do Brasil, Agência Nacional de Vigilância Sanitária (Anvisa) e sociedades de anestesiologia. Incluíram-se também relatos de experiências e consensos de especialistas sobre a temática publicados em periódicos entre janeiro e dezembro de 2020. Essa busca foi realizada no Google Acadêmico e na plataforma de busca da Elsevier, cujo acesso foi disponibilizado de forma aberta durante a pandemia de Covid-19.

$\mathrm{Na}$ segunda etapa, desenvolveu-se o método de treinamento a partir da união dos aspectos teóricos e operacionais próprios da estratégia instrucional em foco, a $\mathrm{PDCR}^{12}$, com as principais evidências e diretrizes encontradas acerca do tema de intubação orotraqueal. Todos os aspectos teórico-operacionais foram baseados no estudo original da $\mathrm{PDCR}^{12}$ e em pesquisas complementares dessa estratégia ${ }^{13,14}$. Destaca-se que a fundamentação desses estudos é pautada nas pesquisas de aquisição de habilidades, expertise e prática deliberada de Ericsson ${ }^{15}$. Em conformidade com a PDCR, criou-se também um guia de aplicação dessa estratégia no contexto estudado.

Este estudo foi desenvolvido em consequência da parceria entre profissionais estudiosos da área de simulação clínica, incluindo a PDCR, e profissionais anestesiologistas que atuam na assistência e/ou docência em um hospital de ensino de referência no Brasil. Ressalta-se que os conteúdos técnicos da intubação orotraqueal foram avaliados e revisados por esses profissionais.

\section{RESULTADOS}

\section{Aspectos teóricos da Prática Deliberada em Ciclos Rápidos}

Para o entendimento da PDCR, é necessário aproximarse do conceito de prática deliberada, cunhado por Ericsson ${ }^{15}$ em estudos sobre aquisição de habilidades e expertise. Esses estudos explicam que as pessoas podem aprimorar sua performance por meio da prática comum ou com a ajuda de terceiros, como professores ou supervisores. Depois, com a experiência adquirida, serão capazes de atuar de forma mais rápida, de modo a alcançar a automatização. No entanto, ao atingirem esse estágio, as pessoas perdem o controle consciente da execução da tarefa, o que as incapacita de realizar melhorias. Para permitir um aperfeiçoamento de performance ultrapassando a linha da automatização, devem, então, realizar a prática deliberada. Essa expressão é definida como uma sequência de atividades específicas cujo objetivo é melhorar o desempenho de indivíduos submetendo-os aos seguintes fatores: 1. tarefa com objetivo definido; 2. motivação para melhorar; 3. feedbacks; e 4. vasta oportunidade de repetição e refinamento gradual de desempenho ${ }^{15}$.

A PDCR se apropria do conceito de prática deliberada, porém utiliza a simulação clínica como base técnica para aplicála. Criada em 2014 por Hunt et al. ${ }^{12}$ em cenário de reanimação cardiopulmonar pediátrica, a PDCR explora um caso clínico incentivando o indivíduo a praticar tarefas até que atinja a maestria. Para tal, inclui uma rápida transição entre essas tarefas e os feedbacks direcionados, fornecidos por um instrutor. Percebe-se, então, que a PDCR mantém os fatores próprios do conceito de prática deliberada, contudo organiza seus próprios aspectos conceituais, denominados de princípios ${ }^{12}$.

O primeiro princípio da PDCR é a maximização do tempo em prática deliberada, a qual significa conceder numerosas oportunidades para a repetição de tarefas. O propósito é atingir o nível de automatização já com maestria, criando memória muscular para fazer o certo. Logo, é necessário receber informações de um instrutor para moldar a habilidade. Essas informações recebidas são denominadas feedbacks, que são fornecidos quando se constata um erro de performance. Esse tipo de feedback é classificado como direcionado e imediato ${ }^{12,13}$.

O segundo princípio é exatamente o fornecimento de feedbacks com evidência. Para que isso seja possível, os instrutores devem possuir domínio do tema tratado na simulação. Devem também ter confiança na aplicação da estratégia de PDCR. Como o próprio nome sugere, o feedback com evidência deve conter informações científicas mais atuais encontradas em literatura ou levar em consideração o consenso de especialistas. Para fornecê-lo, o instrutor fará interrupções breves e assertivas a fim de explicitar claramente a evidência. Não é adequado realizar perguntas ou debates reflexivos durante o treinamento com PDCR. A ideia é que o instrutor oferte a evidência de forma prescritiva, o participante a receba e retorne a exercer a tarefa consertando a ação que gerou o feedback. Em caso de persistência de erro, novo feedback será fornecido, criando-se um rápido ciclo entre tarefa, interrupção e feedback, o que é denominado ciclo feedback-repetição ou ciclo rápido ${ }^{12-14}$.

O terceiro princípio preocupa-se com a qualidade do recebimento dos feedbacks. Foi pensado por conta do possível comportamento defensivo que o participante pode adotar ao ser corrigido sucessivamente. Para evitar essa postura e permitir que as correções sejam aceitas de forma favorável, o terceiro e último princípio é denominado segurança psicológica explícita. Não se inicia o treinamento sem esclarecer que os contínuos feedbacks são essenciais para conquistar uma performance com maestria. É necessário firmar um acordo entre instrutor e participantes a fim de possibilitar um ambiente confortável para o treinamento, garantindo que os participantes estão cientes de que serão interrompidos 
e corrigidos, e vistos da mesma forma como um treinador enxerga um atleta de alto desempenho. Isso caracteriza a dinâmica como um estilo de coaching ${ }^{12,13}$.

\section{Aspectos operacionais da Prática Deliberada em Ciclos Rápidos na intubação orotraqueal}

Em termos de operacionalização para aplicação da PDCR, o número de participantes recomendado em outros estudos é de dois, no mínimo, e entre cinco e seis, no máximo ${ }^{12,13}$. No entanto, esse número tem se mostrado empiricamente variável. Para a intubação orotraqueal no contexto de pandemia por Covid-19, a fim de evitar contaminação pelo novo coronavírus, há a recomendação de que o procedimento seja realizado por dois profissionais: um intubador e um auxiliar ${ }^{16}$. Assim, sugere-se que o treinamento seja realizado em duplas, com um máximo de seis participantes. Enquanto dois estarão realizando as tarefas e recebendo feedbacks, os demais estarão posicionados de forma que possam observar e ouvir os feedbacks dados aos colegas de treinamento, até que haja um revezamento ${ }^{13}$. A dinâmica da troca dos participantes é atrelada às etapas do guia de aplicação.

Respeitando-se os princípios da PDCR, o ato inicial do treinamento deve ter como foco garantir a segurança psicológica12, e, para isso, o treinador explica toda a dinâmica da simulação, destacando os benefícios de uma performance com maestria; nesse caso, evitar a autocontaminação, garantir segurança do paciente e proporcionar ventilação mecânica invasiva de qualidade. Deve-se frisar que, ao assumir o papel de instrutor, o treinador é responsável por ajudar os participantes a realizar as tarefas de forma perfeita, a fim de criarem memória muscular sem erros ${ }^{12}$, resultando no aperfeiçoamento de performance. Toda essa explicação deve ser fornecida de forma carismática e acolhedora. É nesse momento que se declara o acordo pertencente ao estilo coaching ${ }^{12,13}$.

O segundo ato do treinador é apresentar todos os equipamentos disponíveis na simulação e decidir/perguntar qual será a dupla iniciadora ${ }^{13}$. A seguir, narra-se o caso clínico, e temse o início da prática com a dupla escolhida realizando a primeira atividade. Caso haja erro, o treinador interrompe as duplas e fornece o feedback direcionado, prescritivo e imediato ${ }^{12-14}$. Cabe ratificar que o feedback deve conter a evidência científica de forma explícita ${ }^{13}$. Para ilustrar a maneira correta de fornecer um feedback na PDCR, no contexto de intubação orotraqueal, pensou-se no seguinte exemplo: o participante não instalou o filtro High Efficiency Particulate Air (Hepa) entre a máscara e a válvula de Continuous Positive Airway Pressure (Cpap). O treinador deve dizer: "É necessário instalar o filtro Hepa entre a máscara e a válvula de Cpap, de acordo com a Nota técnica n 4 de 2020 da Anvisa para filtrar o vírus e diminuir a chance de contaminação"16. Se o participante cometer o mesmo erro, a interrupção deverá ser da seguinte forma: "Vamos interromper, volte dez segundos e tente novamente"12,13.

A dupla que iniciou o treinamento precisa completar a primeira etapa do guia, de forma perfeita, para que haja o primeiro revezamento. Após a troca, a segunda dupla também deve começar a realizar as tarefas da primeira etapa até concluí-la sem erros. O mesmo procedimento ocorre com a última dupla. Quando todos completarem a primeira etapa, o treinador dará início à segunda etapa e assim sucessivamente, porém, independentemente da etapa treinada, após o revezamento, todo o processo deve sempre começar pela primeira etapa ${ }^{13}$. É recomendável também que a dupla troque os papéis entre si, de modo que ambos possam assumir as funções de intubador e auxiliar.

\section{Guia para aplicação da Prática Deliberada em Ciclos Rápidos}

O guia é composto por tarefas específicas divididas em seis etapas (Quadro 1). A primeira abrange a paramentação, e a segunda diz respeito aos procedimentos iniciais e ao preparo do paciente. A terceira etapa abrange a pré-oxigenação, enquanto a quarta comporta as tarefas de indução em sequência rápida. A quinta etapa refere-se à intubação orotraqueal, e a última abrange a desparamentação. Como cada tarefa do guia foi construída de forma a considerá-la um ponto crítico, nenhuma deve ser ignorada. $\mathrm{O}$ erro de um ponto crítico resultaria em potencial contaminação do profissional e/ou do paciente, assim como interferiria negativamente no cuidado assistencial.

\section{DISCUSSÃO}

Neste estudo, foram apresentadas instruções para a realização de treinamentos com a PDCR para o manuseio de vias aéreas de pacientes hipoxêmicos suspeitos e positivos para Covid-19. Como visto, a PDCR é uma estratégia instrucional de simulação centrada no aprendiz que detecta as falhas na performance do indivíduo e permite consertá-las mediante feedbacks prescritivos, podendo melhorar as deficiências individuais ou de equipe ${ }^{14}$.

Assim, identificam-se situações em que a PDCR pode ser aproveitada para impactar a performance clínica. Essas situações são verdadeiras oportunidades educacionais que devem incluir as seguintes características: diretrizes de desempenho existentes e bem estabelecidas; necessidade do domínio de comportamento; tempo de ensino limitado; eventos de baixo volume, alto risco e urgentes; e situações da equipe que exigem coreografias específicas ou se beneficiam delas ${ }^{14}$. $O$ momento pandêmico atual apresenta essas características, visto que o manuseio de vias aéreas traz um alto risco de contaminação para os profissionais, possui diretrizes estabelecidas e deve ser treinado com domínio de comportamento. 
Quadro 1. Guia para PDCR no manuseio de vias aéreas de pacientes hipoxêmicos suspeitos e positivos para Covid-19.

\section{Etapa 1 - Paramentação}

Realizar paramentação com EPI (ver artigo de PDCR para paramentação e desparamentação ${ }^{13}$ ).

Calçar dois pares de luvas ao entrar no leito.

Checar os EPI por dois profissionais diferentes (intubador e auxiliar).

\section{Etapa 2 - Procedimentos iniciais e preparo do paciente}

Garantir o máximo de dois anestesistas dentro do leito do paciente, e um outro anestesista deve permanecer paramentado do lado de fora para qualquer auxílio.

Checar kit de medicações com as drogas para indução e drogas aspiradas:

- Propofol $160 \mathrm{mg}$ + lidocaína: $80 \mathrm{mg}$ (aspirados juntos em seringa de $20 \mathrm{~mL}$ )

- Fentanil $150 \mathrm{mcg}$

- Succinilcolina $100 \mathrm{mg}$

- Efedrina (solução $10 \mathrm{mg} / \mathrm{mL}$ )

- Atropina (solução $250 \mathrm{mcg} / \mathrm{mL}$ )

- Fenilefrina (solução $40 \mathrm{mcg} / \mathrm{mL}$ )

- Epinefrina (solução $5 \mathrm{mcg} / \mathrm{mL}$ )

- Norepinefrina (solução $16 \mathrm{mcg} / \mathrm{mL}$ )

Checar material de Via Aérea:

- Videolaringoscópio + lâmina,

- Bougie,

- TOT $(5,5-8,5)$, dois de cada,

- Filtros Hepa (3),

- Cânulas de Guedel,

- Cânulas nasofaríngeas,

- Máscaras laríngeas,

- Pinça forte,

- Swivel.

Deixar o TOT montado com swivel e bougie. Colocar tampa vermelha do filtro ocluindo a saída principal do swivel.

Instalar filtro Hepa entre a máscara e a válvula de Cpap (conectada ao sistema Mapleson C) ou entre a máscara, bolsa e válvula (se não houver válvula de Cpap).

Checar com a equipe local se o ventilador mecânico foi testado pelo pessoal do setor (intubador).

Checar se filtro Hepa está instalado entre o ramo expiratório do circuito de ventilação mecânica (auxiliar) e confirmar em voz alta.

Checar todas as extensões do circuito do ventilador (auxiliar) e reforçá-las de forma a evitar desconexões durante o procedimento. Confirmar em voz alta.

Checar conexões do equipamento do sistema Mapleson C e reforçá-las de forma a evitar desconexões durante o procedimento (intubador). Confirmar em voz alta.

Checar se sistema de aspiração está funcionante (intubador) e confirmar em voz alta.

Checar se sistema de fluxo de $\mathrm{O}_{2}$ está funcionante em fluxômetro na saída de gases da parede ou em cilindro de $\mathrm{O}_{2}$ (intubador). Confirmar em voz alta.

Checar a presença de carrinho de parada próximo ao leito (intubador) e confirmar em voz alta.

Checar a presença de desfibrilador e perguntar à equipe local se ele foi testado (intubador).

Checar a presença de duas lixeiras uma de cada lado do leito (auxiliar) e confirmar em voz alta.

Checar a presença e permeabilidade de acesso venoso (auxiliar) com $5 \mathrm{~mL}$ de Soro Fisiológico 0,9\% e confirmar em voz alta.

Conectar TriWays com medicações de indução acopladas (auxiliar) e confirmar em voz alta.

Checar se o paciente está corretamente monitorizado (auxiliar) e confirmar em voz alta.

\section{Etapa 3 - Pré-oxigenação}

Posicionar o paciente para maximizar o tempo de apneia seguro (intubador).

Vedar bem a máscara facial em posição V/E (intubador).

Realizar pré-oxigenação por três a cinco minutos com: $\mathrm{O}_{2} 100 \%$, sempre com filtro Hepa, $2 \mathrm{~L} / \mathrm{min}$ de fluxo de $\mathrm{O}_{2}$, mantendo válvula de Cpap em $5 \mathrm{~cm} \mathrm{H}_{2} \mathrm{O}$ (intubador).

\section{Etapa 4 - Indução em sequência rápida}

Realizar anestesia e relaxamento muscular profundos (intubador solicita e auxiliar administra):

- Fentanil $150 \mathrm{mcg}$ no terceiro minuto da pré-oxigenação,

- Lidocaína: 80 mg + propofol 150 mg, um minuto após o fentanil (lidocaína aspirada com propofol),

- Succinilcolina: $100 \mathrm{mg}$ (ou rocurônio: $100 \mathrm{mg}$ ) assim que o paciente ficar inconsciente. 
Quadro 1. (Continuação) Guia para PDCR no manuseio de vias aéreas de pacientes hipoxêmicos suspeitos e positivos para Covid-19.

Etapa 4 - Indução em sequência rápida

Garantir o bloqueio neuromuscular total antes da tentativa de intubação traqueal.

Não realizar anestesia periglótica.

Se necessário, solicitar a realização de manobras técnicas como Backward Upward Right Pressure (BURP) ao auxiliar (intubador).

Se ventilação manual for necessária:

- $\quad$ utilizar baixos volumes correntes (200-250 mL) sob baixas pressões (15-20 $\mathrm{cmH}_{2} \mathrm{O}$ ),

- adotar preferencialmente ventilação a quatro mãos para evitar vazamentos (pela máscara, ao redor do nariz e queixo),

- utilizar cânula orofaríngea.

\section{Etapa 5 - Intubação orotraqueal}

Realizar a videolaringoscopia (intubador).

Intubar com um tubo traqueal de 7,0-8,0 mm (nas mulheres) ou 8,0-9,0 mm (em homens) (intubador).

Insuflar o cuff ainda visualizando com o videolaringoscópio (auxiliar) e confirmar verbalmente a visualização (intubador e auxiliar).

Descartar a seringa no lixo (auxiliar).

Segurar o TOT próximo à boca (auxiliar), enquanto o intubador recua o bougie segurando próximo à conexão com swivel.

Clampear o TOT com pinça forte (auxiliar).

Clampear o TOT com pinça forte quando o bougie estiver acima da saída do cuff (auxiliar).

Jogar o bougie no lixo (intubador).

Retirar a tampa vermelha do swivel e descartar no lixo (auxiliar).

Conectar o circuito de ventilação (intubador).

Desclampear o TOT (auxiliar) .

Solicitar ao auxiliar que segure/estabilize o TOT para evitar desposicionamento (intubador).

Remover luvas externas e calçar luvas novas (intubador).

Solicitar ao médico assistente ou fisioterapeuta o início da ventilação mecânica (intubador).

Confirmar o IOT com a observação da expansão torácica e $\mathrm{EtCO}_{2}$. Não auscultar (intubador).

Fixar o TOT (intubador).

Colocar a lâmina do videolaringoscópio em uma bolsa plástica com duplo zip lock. Selar a bolsa plástica (auxiliar).

Remover luvas externas e calçar luvas novas (auxiliar).

Se for necessária aspiração traqueal, utilizar sistema fechado.

Administrar midazolan 5 mg, fentanil 100 mcg e rocurônio 50 mg (intubador solicita e auxiliar administra).

Solicitar à equipe médica do local a manutenção da sedação e analgesia com drogas apropriadas, comunicando verbalmente o que é administrado (intubador).

\section{Etapa 6 - Desparamentação}

Realizar desparamentação (ver artigo de PDCR para paramentação e desparamentação' ${ }^{13}$ ).

EPI: equipamentos de proteção individual; Hepa: High Efficiency Particulate Air; TOT: tubo orotraqueal; IOT: intubação orotraqueal.

Diversos estudos ressaltam os benefícios dos treinamentos com PDCR, a maioria mostrando melhorias no desempenho nas reanimações cardiopulmonares ${ }^{12,17,18}$, revelando resultados de compressões de maior qualidade, tempo mais rápido para iniciar compressões e desfibrilação, inclusive comparando com os treinamentos padrão da American Heart Association ${ }^{17,18}$. Outras melhorias observadas são referentes à comunicação da equipe e ao gerenciamento das vias aéreas ${ }^{17}$. Nessa perspectiva, um estudo de treinamento com PDCR para coreografia de intubação mostra uma pontuação dos residentes significativamente mais alta em lista de verificação de intubação comparados aos que receberam feedback pós-cenário. O estudo sugere que a PDCR é uma estratégia eficaz para ensinar a habilidade de intubação, enfatizando a coreografia do procedimento ${ }^{19}$.

Serão discutidos, a seguir, de maneira sintética, alguns aspectos técnicos adotados para a construção do guia para aplicação de PDCR, visto que ele deve ser construído de acordo com a realidade da instituição quanto aos recursos humanos e materiais.

O preparo minucioso para a realização do procedimento minimiza as chances de infecção cruzada e otimiza as chances de uma intubação efetiva e segura. Nenhuma falha ou imperfeição é mínima quando há risco para os pacientes e profissionais 
envolvidos. Por isso, indica-se no guia a dupla checagem de forma sistemática dos recursos humanos e materiais necessários para a realização da intubação traqueal, além da confirmação verbal das ações realizadas, com o objetivo de garantir uma comunicação efetiva e segura ${ }^{11,20}$. Além disso, indica-se que o plano de abordagem para o manuseio de via aérea, incluindo planos alternativos, deve ser repassado antes do procedimento, e todos devem estar de acordo ${ }^{8}$.

O uso de EPI, embora sejam indispensáveis, torna a performance do procedimento muitas vezes dificultada, o que pode comprometer a efetividade do processo. $O$ uso da máscara $\mathrm{N}-95$, óculos de proteção, protetor facial (face shield), capote e outros materiais atrapalha a comunicação e a visualização, dificultando a execução da intubação. Estudo recente apontou que 11 profissionais reportaram visualização dificultada por embaçamento nas máscaras ${ }^{8,10,16}$.

Indica-se que para a intubação estejam presentes dois profissionais: o profissional mais habilitado deve realizar o procedimento com auxílio de um segundo operador, com o intuito de otimizar o sucesso na primeira tentativa. Um terceiro participante pode ficar de prontidão como assistente e, caso necessário, ser solicitado ${ }^{8,16}$.

A técnica de intubação indicada é a de sequência rápida na tentativa de evitar o uso de ventilação bolsa máscara e otimizar a sedação/analgesia/bloqueio neuromuscular. A ventilação com máscara bolsa válvula antes da intubação é considerada um procedimento de alto risco para gerar aerossóis, assim como uma sedação inadequada poderá colocar o intubador em risco se o paciente tossir e ficar agitado, podendo, inclusive, correr o risco de desalojar o $\mathrm{EPI}^{10,11,20-23}$.

O uso de videolaringoscópio é recomendado em detrimento da laringoscopia tradicional, com o objetivo de otimizar o sucesso do procedimento na primeira tentativa ${ }^{10,11,20}$. No guia, apresenta-se como estratégia a intubação com uso de um introdutor (bougie) através do swivel, uma vez que funcionaria como vedação efetiva contra os aerossóis.

Recomenda-se a pré-oxigenação por três a cinco minutos para otimizar as condições para o procedimento, se possível com elevação da cabeceira, no entanto faltam evidências na literatura quanto ao dispositivo ideal para a realização da préoxigenação $8,10,11,20$. Propõe-se nesse guia de treinamento a utilização da válvula de Cpap acoplada a um sistema Mapleson $C$, que parece ser efetiva na pré-oxigenação dos pacientes, mesmo naqueles inicialmente muito hipoxêmicos e obesos.

\section{CONCLUSÃO}

Neste artigo, aspectos teóricos, conceituais e instruções operacionais detalhadas de aplicação da PDCR são apresentados a fim de instrumentalizar os serviços de saúde na realização de treinamentos. Essa estratégia instrucional parece ser propícia a treinar com maestria os profissionais que realizarão o procedimento de intubação orotraqueal no enfrentamento das formas graves da Covid-19, visando minimizar o risco de contaminação. Essa iniciativa vai ao encontro do incentivo dos autores criadores da estratégia de PDCR em aplicá-la em contextos diferentes do original, de reanimação cardiopulmonar, objetivando testar a sua aplicabilidade em outras conjunturas e instituições. Além disso, proporciona a incorporação do treinamento em serviço, com ênfase no binômio teoria e prática baseada em evidência.

O artigo limitou-se à etapa de criação do guia de aplicação da PDCR, além de dar subsídios teóricos e operacionais para a aplicabilidade. No entanto, indica-se que o guia seja validado por especialistas, e sugere-se que estudos clínicos sejam realizados para gerar resultados concretos sobre a aplicabilidade da PDCR nesse cenário. Salienta-se que ajustes podem ser realizados nesse guia tanto para atender à realidade das instituições quanto a atualizações de evidências/recomendações.

\section{CONTRIBUIÇÃO DOS AUTORES}

Rodrigo Pereira Diaz André participou da concepção e do desenho do estudo, e da redação do manuscrito, sendo responsável pela escrita dos aspectos concernentes à intubação orotraqueal. Hudson Carmo de Oliveira participou da concepção e do desenho do estudo, e da redação do manuscrito, sendo responsável pela escrita dos aspectos concernentes à Prática Deliberada em Ciclos Rápidos. Glauber Gouvêa participou da análise e interpretação de dados, e da revisão crítica do conteúdo intelectual importante, sendo responsável pela revisão do conteúdo da intubação orotraqueal. Fátima Carneiro Fernandes participou da análise e interpretação de dados, e da revisão crítica do conteúdo intelectual importante, sendo responsável pela revisão total do artigo. Ingrid Régia Lopes Jerônimo participou da análise e interpretação de dados, e da revisão crítica do conteúdo intelectual importante, sendo responsável pela revisão do conteúdo da Prática Deliberada em Ciclos Rápidos. Juliana Faria Campos participou da concepção e do desenho do estudo, e da redação do manuscrito, sendo responsável pela problematização e esquematização do artigo em geral e pela revisão crítica do conteúdo intelectual importante, além de atuar como orientadora e revisora geral do texto.

\section{CONFLITO DE INTERESSES}

Declaramos não haver conflito de interesses.

\section{FINANCIAMENTO}

Coordenação de Aperfeiçoamento de Pessoal de Nível Superior (Capes) - Ministério da Educação. Autor Hudson Carmo de 
Oliveira. Programa de excelência acadêmica. Processo $\mathrm{n}^{\circ}$ 88887.471399/2019-00.

\section{REFERÊNCIAS}

1. World Health Organization. Coronavirus disease 2019 (Covid-19) Situation report no 117 [access in 17 may 2020]. Available from: https://www.who. int/docs/default-source/coronaviruse/situation-reports/20200516-covid19-sitrep-117.pdf?sfvrsn=8f562cc_2.

2. Guan WJ, Ni ZY, Hu Y, Liang WH, Ou CQ, He JX, et al. Clinical characteristics of coronavirus disease 2019 in China. N Engl J Med. 2020;382:1708-20. doi: 10.1056/NEJMoa2002032.

3. Wang D, Hu B, Hu C, Zhu F, Liu X, Zhang J, et al. Clinical characteristics of 138 hospitalized patients with 2019 novel coronavirus-infected pneumonia in Wuhan, China. JAMA. 2020;323(11):1061-9. doi: 10.1001/jama.2020.1585.

4. Yang X, Yu Y, Xu J, Shu H, Xia J, Liu H, et al. Clinical course and outcomes of critically ill patients with Sars-CoV-2 pneumonia in Wuhan, China: a single-centered, retrospective, observational study. Lancet Respir Med. 2020;8(5):475-81. doi:10.1016/S2213-2600(20)30079-5.

5. Tran K, Cimon K, Severn M, Pessoa-Silva CL, Conly J. Aerosol generating procedures and risk of transmission of acute respiratory infections to healthcare workers: a systematic review. PLoS One. 2012;7(4):e35797.

6. Chinese Society of Anesthesiology, Chinese Association of Anesthesiologists. Perioperative management of patients infected with the novel coronavirus. Anesthesiology. 2020;132:1307-16.

7. World Health Organization. Summary of probable Sars cases with onset of illness from 1 November 2002 to 31 July 2003 [access in 7 feb 2020]. Available from: https://www.who.int/csr/sars/ country/table2004_04_21/en.

8. Yao W, Wang T, Jiang B, Gao F, Wang L, Zheng H, et al. Emergency tracheal intubation in 202 patients with Covid-19 in Wuhan, China: lessons learnt and international expert recommendations. $\mathrm{Br} J$ Anaesth. 2020;125(1):e28ee37.

9. Gonzalez L, Kardong-Edgren S. Deliberate practice for mastery learning in nursing. Clin Simul Nurs. 2016;13(1):10-4. doi: 10.1016/j.ecns.2016.10.005.

10. Chen X, Liu Y, Gong Y, Guo X, Zuo M, Li J, et al. Perioperative Management of patients infected with the novel coronavirus recommendation from the joint task force of the Chinese Society of Anesthesiology and the Chinese Association of Anesthesiologists. Anesthesiology 2020;132(6):1307-16. doi: 10.1097/ALN.0000000000003301.

11. Anaesthesia and caring for patients during the Covid-19 outbreak. Australian Society of Anaesthetists. For the latest version, please visit https://asa.org.au/covid-19-updates/.

12. Hunt EA, Duval-Arnould JM, Nelson-McMillan KL, Bradshaw JH, Diener-West M, Perretta JS, et al. Pediatric resident resuscitation skills improve after "Rapid Cycle Deliberate Practice" training. Resuscitation. 2014;85(7):945-51.
13. Oliveira HC, Souza LC, Leite TC, Campos JF. Personal protective equipment in the coronavirus pandemic: training with Rapid Cycle Deliberate Practice. Rev Bras Enferm. 2020;73(Suppl 2):e20200303. doi: 10.1590/0034-71672020-0303.

14. Perretta JS, Duval-Arnould J, Poling S, Sullivan N, Jeffers JM, Hunt EA, et al. Best practices and theoretical foundations for simulation instruction using Rapid-Cycle Deliberate Practice. Simul Healthc. 2020;15(5):356-62. doi: 10.1097/SIH.0000000000000433.

15. Ericsson KA. Deliberate practice and acquisition of expert performance: a general overview. Acad Emerg Med. 2008;15(11):988-94.

16. Agência Nacional de Vigilância Sanitária. Nota Técnica nº4/2020. Orientações para serviços de saúde: medidas de prevenção e controle que devem ser adotadas durante a assistência aos casos suspeitos ou confirmados de infecção pelo novo coronavírus (Sars-CoV-2). Brasília: Anvisa; 2020.

17. Hunt E, Duval-Arnould J, Chime N, Jones K, Rosen M, Hollingsworth $M$, et al. Integration of in-hospital cardiac arrest contextual curriculum into a basic life support course: a randomized, controlled simulation study. Resuscitation. 2017;114(5):127-32.

18. Sullivan N, Duval-Arnould J, Twilley M, Smith SP, Aksamit D, Boone-Guercio $P$, et al. Simulation exercise to improve retention of cardiopulmonary resuscitation priorities for in-hospital cardiac arrests: a randomized controlled trial. Resuscitation. 2015;86(1):6-13.

19. Gross I, Abrahan D, Kumar A, Noether J, Shilkofski NA, Pell P, et al. Rapid Cycle Deliberate Practice (RCDP) as a method to improve airway management skills: a randomized controlled simulation study. Cureus 2019;11(9):e5546.

20. Wax RS, Christian MD. Practical recommendations for critical care and anesthesiology teams caring for novel coronavirus (2019-nCoV) patients. Can J Anesth. 2020;67:568-76. doi: 10.1007/s12630-020-01591-x.

21. World Health Organization. Novel coronavirus (2019-nCoV) technical guidance: infection prevention and control. WHO, 2020. [access in 9 feb 2020]. Available from: https://www.who.int/emergencies/diseases/novelcoronavirus-2019/technical-guidance/infection- prevention-and-control.

22. Centers for Disease Control and Prevention. Interim infection prevention and control recommendations for patients with confirmed 2019 novel coronavirus (2019-nCoV) or persons un- der investigation for 2019 $\mathrm{nCoV}$ in healthcare settings. Centers for Disease Control and Prevention, 2020. [access in 9 feb 2020]. Available from: https://www.cdc.gov/ coronavirus/2019-nCoV/ hcp/infection-control.html.

23. University of Toronto. Coronavirus and safety precaution. University of Toronto, 2020. [access in 9 feb 2020]. Available from: https://www. anesthesia.utoronto.ca/news/coronavirus-and-safety-precautions. 